COMMENT. Intracranial hypotension and Marfan syndrome. A PubMed search found several single case reports of spontaneous intracranial hypotension in children, two with Marfan syndrome. A 14-year-old girl followed for Marfan syndrome presented with a postural headache for a month, and MRI showed bilateral subdural hematomas. Large lumbosacral arachnoid diverticula were shown on spinal MRI. Headaches resolved after epidural blood patching. Dural ectasia is a common finding with Marfan syndrome. (Cheuret E, et al. Childs Nerv Syst 2008 Apr;24(4):509-13).

A 12-year-old girl with Marfan syndrome, sacral dural ectasia, and tonsillar herniation, presented with headache, unresponsive to surgical decompression of the foramen magnum. Further MR imaging demonstrated CSF leak at the level of the dural ectasia, and the headache resolved after blood patch of the leak. (Puget S, et al. J Neurosurg 2007 Jan;106(1 Supp1):48-52).

Skull thickening, paranasal sinus expansion, and sella turcica shrinkage are reported in a 29-year-old man with a history of VP shunt placement following traumatic brain injury at 9 years of age. MRI showed signs of chronic intracranial hypotension, and LP opening pressure was not recordable. Secondary installation of a valve to restore normal ICP is recommended. (Yoon MK, et al. J Neurosurg Pediatr 2013 Jun;11(6):667-72).

\title{
PSEUDOTUMOR CEREBRI SYNDROME
}

Investigators from the University of Texas Southwestern Medical Center, Dallas, and centers in Philadelphia and Salt Lake City, propose updated criteria for the diagnosis of pseudotumor cerebri (PTCS) and its variations. Idiopathic intracranial hypertension (IIH) is an appropriate term for a subset of patients with primary IH of unclear etiology, but not for those precipitated by an identifiable secondary cause. The syndrome is best described using the umbrella term PTCS. Required criteria for the diagnosis of PTCS are as follows: a) Papilledema, b) Normal neurologic examination (except cranial nerves), c) Normal MRI (or CT), d) Normal CSF composition, and e) Elevated lumbar puncture opening pressure $(>250 \mathrm{~mm}$ for adults; $>280 \mathrm{~mm}$ for children [250 if not sedated and not obese]).

With no papilledema, diagnosis requires (b) to (e) satisfied plus abducens nerve palsy.

Uncommon manifestations include a facial nerve palsy, hemifacial spasm, or radicular pain. CSF rhinorrhea or otorrhea and confirmation of a CSF leak are highly suggestive of PTCS diagnosis. Papilledema may be absent in recurrent PTCS cases because of gliosis in the nerve or optic atrophy.

Neuroimaging abnormalities highly suggestive of PTCS are: 1) Empty sella. 2) Flattening of posterior aspect of the globe, 3) Distension of the perioptic subarachnoid space, and 4) Transverse venous sinus stenosis. Tonsillar ectopia is more frequent in cases of PTCS but is not specific; it may be a sign of low CSF pressure, and may indicate an increased risk for herniation with LP. (Friedman DI, Liu GT, Digre KB. Revised diagnostic criteria for the pseudotumor cerebri syndrome in adults and children. Neurology 2013 Sep 24;81(13):1159-65). (Response: Dr Friedman, E-mail: Deborah.Friedman@UTSouthwestern.edu). 
COMMENT. Present diagnostic criteria and guidelines for the management of pediatric PTC do not consider pediatric aspects, according to a study involving all pediatric hospitals in Germany (Tibussek D, et al. Klin Padiatr 2013 Mar;225(2):81-5). The annual incidence was 0.5 per 100,000 children. A wide range of vision problems included papilledema, visual loss, diplopia, visual field defect, disturbed color and stereo vision. Papilledema was absent in $10(16.4 \%)$ of a total of 61 patients treated Jan to Dec 2008. The importance of the ophthalmological exam was emphasized.

In a study of 42 patients with average age at onset of 10.8 years (range, 12 months to 17 years), obesity was found in $11(26 \%)$. Headache occurred in $76 \%$. Various etiologic factors were associated, including trauma in 2, hypervitaminosis A, corticosteroid withdrawal, oral contraceptives, Guillain-Barre syndrome, urinary tract infection, varicella-zoster virus infection, and dural venous sinus thrombosis associated with otitis media. Prompt diagnosis and medical treatment are important to avoid visual loss. (Per H, et al. Brain Dev 2013 Jun;35(6):561-8).

An unusual case of a 13-year-old boy presented with acute paresis of the left abducens, facial and vagus nerves. Brain MRI and angiography were normal. LP revealed an intracranial pressure of $575 \mathrm{mmH} 2 \mathrm{O}$. Treatment with acetazolamide resulted in improvement with no sequelae (Antoun J et al. J Fr Ophthalmol 2013 May 31 [Epub ahead of print]).

\section{DEVELOPMENTAL MALFORMATIONS}

\section{WILLIAMS-BEUREN SYNDROME WITH BRAIN DYSPLASIA}

Investigators from Jichi and Yokohama City Universities, Japan, report a patient with the common Williams-Beuren syndrome (WBS) deletion in 7q11.23 who presented with severe cerebral and cerebellar dysplasia and progressive hypertrophic cardiomyopathy. Facial characteristics included downward slanting of the palpebral fissure, blepharophimosis, strabismus, right iris hypopigmentation, low-set ears, higharched palate, broad nose, thick lips, wide mouth, and micrognathia. Associated abnormalities included a deep, husky voice, hearing impairment by brainstem auditory responses, hypoplastic toes with nail aplasia, and contractures of hip and knee joints. Progressive hypertrophic cardiomyopathy manifested with ventricular septal defect at birth, right ventricular hypertrophy at 1 month, and left ventricular hypertrophy and mitral valve insufficiency at age 2 months. Supravalvular aortic stenosis commonly found with WB syndrome was absent.

Brain MRI showed congenital hydrocephalus, hypoplasia of the cerebellum and brain stem, and agenesis of the corpus callosum. At 3 months of age, he developed recurrent generalized tonic convulsions daily. Laboratory findings included mild hypocalcemia, low blood sugar, and low free T4 and high TSH consistent with hypothyroidism. The patient died with heart failure at age 1 year 5 months. (Okamoto $\mathrm{N}$, Yamagata T, Yada Y, et al. Williams-Beuren syndrome with brain malformation and hypertrophic cardiomyopathy. Brain Dev 2013 Jul 27 [Epub ahead of print]). (Response: Dr Takanori Yamagata, Department of Pediatrics, Jichi Medical University, Tochigi, Japan. E-mail: takanori@jichi.ac.jp). 